Check for updates

Cite this: RSC Adv., 2019, 9, 9198

\title{
Wash-stable, oxidation resistant conductive cotton electrodes for wearable electronics
}

\author{
Sompit Wanwong, (D) *a Weradesh Sangkhun, (D) ${ }^{\mathrm{a}}$ S. Zohreh Homayounfar, (D) ${ }^{\mathrm{b}}$ \\ Kwang-Won Park (D) ${ }^{b}$ and Trisha L. Andrew (DD *b
}

Commercial, untreated cotton fabrics have been directly silver coated using one-step electroless deposition and, subsequently, conformally encapsulated with a thin layer of poly(perfluorodecylacrylate) (PFDA) using initiated chemical vapor deposition (iCVD). The surface of these PFDA encapsulated fabrics are notably water-repellent while still displaying a surface resistance as low as $0.2 \Omega \mathrm{cm}^{-1}$, making them suitable for incorporation into launderable wearable electronics. X-ray photoelectron spectroscopy confirms that the PFDA encapsulation prevents oxidation of the silver coating, whereas unencapsulated samples display detrimental silver oxidation after a month of air exposure. The wash stability of PFDAencapsulated, silver-coated cotton is evaluated using accelerated laundering conditions, following established AATCC protocols, and the samples are observed to withstand up to twenty home laundering cycles without notable mechanical degradation of the vapor-deposited PFDA encapsulation. As a proofof-concept, PFDA-Ag cotton is employed as a top and bottom electrode in a layered, all-fabric triboelectric generator that produces voltage outputs as high as $25 \mathrm{~V}$ with small touch actions, such as tapping.

Received 4th February 2019

Accepted 14th March 2019

DOI: 10.1039/c9ra00932a

rsc.li/rsc-advances pretreatments, such as chemical etching, coating with adhesive polymers or impregnation with nucleation directors, to encourage metal nano- or microparticle binding on the cotton surface. For example, Ashayer-Soltani et al. reported that a pol$y$ (diallyldimethylammonium chloride) (PDAC) adhesive layer increased the uniformity of the silver particle coating on the surface of prewoven cotton. ${ }^{15}$ Tan et al. impregnated tin(II) chloride into cotton before performing ELD so that the $\mathrm{Sn}^{2+}$ center acted as nucleation site for silver nanoparticles. ${ }^{16}$ The drawbacks here are that the materials best suited for these extra layers (PDAC, $\mathrm{Sn}^{2+}$ ) are often toxic or non-biocompatible, precluding any conductive cotton fabrics thus obtained from being used in wearable electronics.

Another major issue is the laundering stability of silvercoated fabrics, especially silver-coated cotton. While silvercoated synthetic fabrics retain their surface resistivities when exposed to humidity, silver and other metal-coated fabrics quickly form a resistive oxide layer upon being simultaneously exposed to heat and moisture (such as sweat from a human body). Counterproductively, most known encapsulation approaches exponentially increase the surface resistivity of silver-coated synthetics. The rough surface of cotton, in particular, encourages delamination and oxidation of loosely-adhered silver coatings upon exposure to moisture or humidity.

Here, we describe an approach to create water-repellant conductive cotton using a direct, two-step coating and encapsulation procedure. First, we perform a facile electroless deposition on untreated cotton using Tollens' reagent. Next, we
${ }^{a}$ Division of Materials Technology, School of Energy, Environment and Materials, King Mongkut's University of Technology Thonburi, 126 Pracha Uthit Road, Bang Mod, Thung Khru, Bangkok 10140, Thailand. E-mail: sompit.wan@kmutt.ac.th

${ }^{b}$ Department of Chemistry, University of Massachusetts Amherst, Amherst, MA 01003, USA.E-mail: tandrew@umass.edu 
encapsulate the silver coating with a conformal layer of poly(perfluorododecylacrylate) (PFDA) using initiated chemical vapor deposition (iCVD). ${ }^{17}$ Encapsulated samples (PFDA-Ag cotton) retain a very low surface resistance of $0.2 \Omega \mathrm{cm}^{-1}$. As a proof-of-concept, we incorporate PFDA-Ag cotton into an allfabric triboelectric generator (TEG), an energy harvesting device that can convert mechanical energy into electrical power by using the triboelectric effect between positive and negative triboelectric layers.

\section{Experimental sections}

\subsection{Materials}

Silver nitrate $\left(\mathrm{AgNO}_{3}, 99 \%\right)$, ammonium hydroxide $\left(\mathrm{NH}_{4} \mathrm{OH}\right.$, $\left.28 \% \mathrm{NH}_{3}\right)$, dextrose, trichloro $(1 \mathrm{H}, 1 \mathrm{H}, 2 \mathrm{H}, 2 \mathrm{H}$-perfluorooctyl) silane (97\%), $(1 H, 1 H, 2 H, 2 H$-perfluorodecyl)acrylate $(97 \%)$, and tert-butyl peroxide (98\%) were purchased from Sigma Aldrich and used without further purification. Potassium hydroxide $(\mathrm{KOH})$ was purchased from Fisher Scientific. Cotton (combed cotton PFD 108") was purchased from Dharma Trading Co. The cotton fabrics were cut into $1 \times 1$ inch $^{2}$ swatches and were cleaned by soaking in commercial laundry detergent for 30 minutes and washing with copious amounts of DI water before use.

\subsection{Electroless deposition}

Tollens' reagent was prepared by mixing $0.5 \mathrm{M} \mathrm{AgNO}_{3}, 0.6 \mathrm{M}$ $\mathrm{KOH}$ and concentrated $\mathrm{NH}_{4} \mathrm{OH}$ in a $1: 0.05: 0.12$ volume ratio and stirring until the solution turned clear. In a separate vial, Tollens' reagent and $0.5 \mathrm{M}$ aqueous dextrose were mixed in $1: 1$ volume ratio, cotton swatches immersed in this mixture, the vial capped and then sonicated in an ultrasonic bath sonicator for different periods of time (15 $\mathrm{min}, 30 \mathrm{~min}, 60 \mathrm{~min}$ and 120 min). Next, the cotton fabrics were rinsed with copious amounts of deionized water and air-dried overnight.

\subsection{Initiated chemical vapor deposition (iCVD)}

iCVD is a technique that creates polymer films on arbitrary substrates/surfaces using reactive precursors in the vapor phase. For this work, coatings of the fluorinated polymer PFDA were directly created on silverized cotton cloths by effecting the chain growth polymerization of $1 H, 1 H, 2 H, 2 H$-perfluorodecyl acrylate (monomer) inside a low pressure reactor. tert-Butyl peroxide was used as an initiator. The deposition was conducted in a custom-built circular reactor $(290 \mathrm{~mm}$ diameter, $70 \mathrm{~mm}$ height), the approximate design of which is previously reported. ${ }^{17}$ The major components of our reactor included a water-cooled substrate stage, monomer and initiator inlets, and a metal filament heater used to decompose the initiator and create reactive radical species. The liquid monomer, $1 H, 1 H, 2 H, 2 H$-perfluorodecyl acrylate, was placed in an ampule and vaporized by heating to $80{ }^{\circ} \mathrm{C}$. tert-Butyl peroxide was placed in an ampule held at room temperature. The filament heater was maintained at $300{ }^{\circ} \mathrm{C}$ and the substrate stage was maintained at $15{ }^{\circ} \mathrm{C}$. PFDA film growth was allowed to proceed for 20 minutes after monomer and initiator vapors were introduced into the reactor. The reactor pressure was maintained at 400 mTorr pressure throughout the deposition.

\subsection{Preparation of triboelectric materials}

2.4.1 Polydimethylsiloxane film on PFDA-Ag-cotton. Polydimethylsiloxane (PDMS) elastomer was prepared by mixing Dow Corning Sylgard 184 at a base : crosslinker ratio of $10: 1$. This mixture was applied onto the PFDA encapsulated silver coated cotton masked with Kapton tape (thickness of $0.1 \mathrm{~cm}$ ) using doctor blading. The PDMS film thus created was cured at $70{ }^{\circ} \mathrm{C}$ for 2 hours.

2.4.2 Fluoroalkylated cotton (F-cotton). Cotton $(1 \times 1$ inch $\left.^{2}\right)$ were soaked in trichloro $(1 H, 1 H, 2 H, 2 H$-perfluorooctyl) silane/hexanes $(1: 100 \mathrm{v} / \mathrm{v})$ for 30 minutes, followed by rinsing in hexanes and drying under ambient conditions. Functionalization was confirmed using X-ray photoelectron spectroscopy (XPS). After functionalization, F-cotton showed an $\mathrm{F}$ 1s peak at $687.7 \mathrm{eV}$ and an $\mathrm{O} 1 \mathrm{~s}$ peak at $531.3 \mathrm{eV}$. The $\mathrm{C} 1 \mathrm{~s}$ peak of pristine cotton splits into two peaks in the XPS spectrum of F-cotton because of the interaction between fluorine and carbon atoms. ${ }^{18}$

\subsection{Fabrication of triboelectric generator (TEG)}

TEG was fabricated in a face-to-face configuration. The PDMS layer coated onto the Ag cotton was served as the bottom positive layer and the fluoroalkylated cotton (F-cotton) taped with the PFDA encapsulated silver coated cotton was served as the top negative layer.

\subsection{Instrumentation}

Surface resistances were measured using a multimeter (Fluke 27 II) at room temperature. The reported resistances were the average values obtained by measuring five randomly selected positions (the distance between the measurement positions was maintained at $1 \mathrm{~cm}$ ). Surface sheet resistances were measured using a 4-point probe (LUCAS LABS Pro4) connected to a source meter (Keithley 2400). The error of conductivity values corresponds to the standard deviation of the resistivity values for the different data sets. The crystal structure of the silver coated on cotton was investigated using an X-ray diffractometer (XRD) (PANalytical X'Pert Pro). SEM images were obtained using FEI Magellen 400 XHR-SEM. Contact angles were measured by static optical contact angle meter (KINO SL150E). X-ray photoelectron spectroscopy (XPS) (AXIS Ultra DLD) was used to study chemical composition of the Ag-cotton, PFDA-Ag-cotton and fluorooctylsiloxane grafted cotton surface. Laundering stability of PFDA-Ag-cotton was tested according to American Association of Textile Chemists and Colorists (AATCC) test method 61, condition 2A. The PFDA-Ag-cotton was washed in a rotating closed canister containing an aqueous solution of "Without Optical Brightener" (WOB) detergent $(0.15 \% \mathrm{w} / \mathrm{w})$ and 50 stainless steel balls ( $6 \mathrm{~mm}$ diameter). The canister was maintained at a temperature of $49^{\circ} \mathrm{C}$. The voltage and current output of TEG were measured using an oscilloscope (RIGOL DS1104) and source meter (Keithley 2400), respectively. 


\section{Results and discussion}

\subsection{Electroless deposition of silver on cotton and surface} resistance

Electroless deposition of silver onto untreated cotton was accomplished using the Tollens' reaction. First, a freshly-made solution of $\mathrm{AgNO}_{3}$ was mixed with $\mathrm{KOH}$ and $\mathrm{NH}_{4} \mathrm{OH}$ to generate amminated silver ions, which could then be reduced by solubilized dextrose to form silver microparticles $\left(\mathrm{Ag}^{0}\right)$ directly on the cotton surface. Samples were exposed to constant lowintensity sonication as the Tollens' reaction progressed to ensure that the size of the actively-forming silver aggregates remained small minimally disperse. The total deposition time was varied from $15 \mathrm{~min}, 30 \mathrm{~min}, 60 \mathrm{~min}$ or $120 \mathrm{~min}$. As shown in Table 1 and Fig. 1a, the cotton fabric linearly increased in weight, proportional to the reaction time, with a maximum weight gain of $116 \%$ after 120 minutes. A surface resistance of $0.80 \Omega \mathrm{cm}^{-1}$ was achieved after a 15 minute deposition time, which reduced to $0.22 \Omega \mathrm{cm}^{-1}$ after 120 minutes. The small changes in surface resistance at longer deposition times suggested that most of the silver salt $\left(\mathrm{Ag}^{+}\right)$was reduced after 15 minutes. In addition, the surface sheet resistance (conductivity) of the Ag-cotton was measured using a four-point probe and found to be consistent with the aforementioned surface resistance measurements. The amount of electrical current that could be supported by the silver coating (15 minute deposition time) was qualitatively demonstrated by passing current from a $9 \mathrm{~V}$ battery through the fabric to an LED array (Fig. 1b).

\subsection{X-ray diffraction pattern of silver coated cotton}

The X-ray diffraction spectrum (Fig. 2) of silver-coated cotton displayed four peaks at $2 \theta$ angles of $38.2^{\circ}, 44.4^{\circ}, 64.6^{\circ}$, and $77.5^{\circ}$ (labelled (111), (200), (220) and (311), respectively), which are characteristic of face-centered cubic (fcc) silver (JCPDS file no. 04-0783). No other peaks, such as those arising from silver oxide, were detected, confirming that electroless deposition yielded a high purity silver coating. The average size of Ag aggregates was inferred to be $26 \mathrm{~nm}$ using the Debye-Scherrer formula. ${ }^{19,20}$

\subsection{Surface morphologies of silver coated cotton}

Scanning electron micrographs (SEMs) of the silver coated cotton fabrics revealed that the silver aggregates conformally coated the circumference of each fiber of the constituent yarn of the fabric (Fig. 3). With increasing deposition time, however,

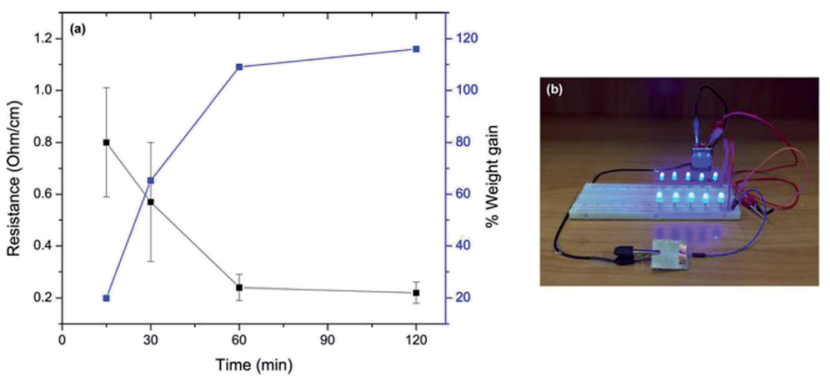

Fig. 1 (a) The relationship between the surface resistance, weight gain and electroless deposition time; (b) photograph of the Ag-cotton placed in a circuit to power LEDs.

the surfaces of the fibers were observed to grow rougher and disordered silver fingers that bridged the gap across multiple fibers were noticed. These brittle features can crack or delaminate from the surface of the fabrics and lead to increased surface resistances with wear. Moreover, oxidation events originate from rough, terraced edges in the presence of heat, oxygen and water and, therefore, such surface roughness had negative implications for the longevity of the silver coatings.

\subsection{Encapsulation silver coated cotton with PFDA, oxidation resistant and laundering stability}

To prevent detrimental oxidation and mechanical washing of thick coatings, we conformally encapsulated the silver coating with a perfluorinated acrylate polymer, PFDA, to increase its lifetime and protect its electrical properties. We chose pol$\mathrm{y}(1 H, 1 H, 2 H, 2 H$-perfluorodecyl acrylate) (PFDA) as the protective layer because fluorinated coatings are hydrophobic and thermally stable (even flame retardant, in some cases). ${ }^{21-24}$ PFDA was applied onto the surface of silver coated cotton using a solvent-free initiated chemical vapor deposition (iCVD) process. ${ }^{17}$ iCVD affords conformal polymer films on topographically complex surfaces. Since this process combines polymer synthesis and deposition into a single step, exquisite control over film thickness and coating uniformity could be achieved. For this work, the PFDA coating thickness (as controlled by varying the deposition time) was approximately $50 \mathrm{~nm}$. After PFDA coating, a droplet of water placed on the surface of the fabric was not observed to penetrate into the fabric (Fig. 4a). The apparent water contact angle on this rough surface was measured to be $134^{\circ}$, indicating that the fabric surface became hydrophobic (Fig. 4b). Because a thin,

Table 1 Surface resistance and \% weight gain of Ag cotton at different deposition time

\begin{tabular}{lllll}
\hline Sample & Deposition time (min) & Weight gain $(\%)$ & Surface resistance $^{a}\left(\Omega \mathrm{cm}^{-1}\right)$ & Surface sheet resistance $^{b}(\Omega$ per square $)$ \\
\hline Cotton & 15 & 20 & $0.80 \pm 0.21$ & $0.39 \pm 0.13$ \\
Cotton & 30 & 65 & $0.57 \pm 0.23$ & $0.15 \pm 0.01$ \\
Cotton & 60 & 110 & $0.24 \pm 0.05$ & $0.07 \pm 0.03$ \\
Cotton & 120 & 116 & $0.22 \pm 0.04$ & $0.01 \pm 0.005$
\end{tabular}

${ }^{a}$ Measured by a multimeter. ${ }^{b}$ Measured by 4 -point probe. 


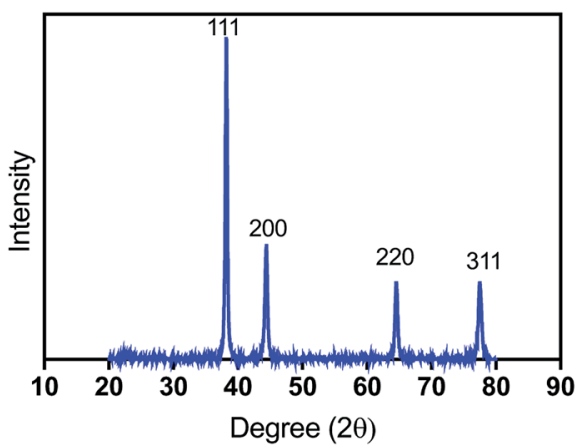

Fig. 2 XRD spectrum of Ag deposited on cotton.
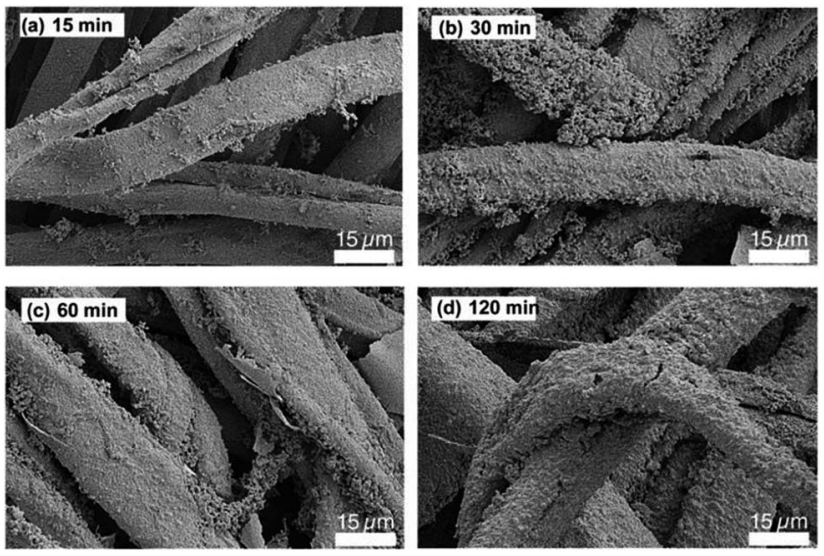

Fig. 3 (a)-(d) SEM images of Ag coated cotton with increasing of deposition time.

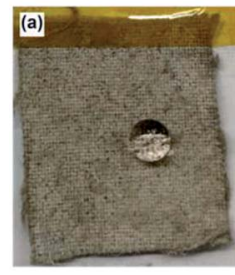

(b)

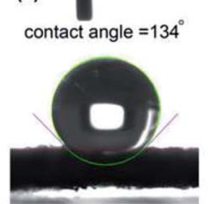

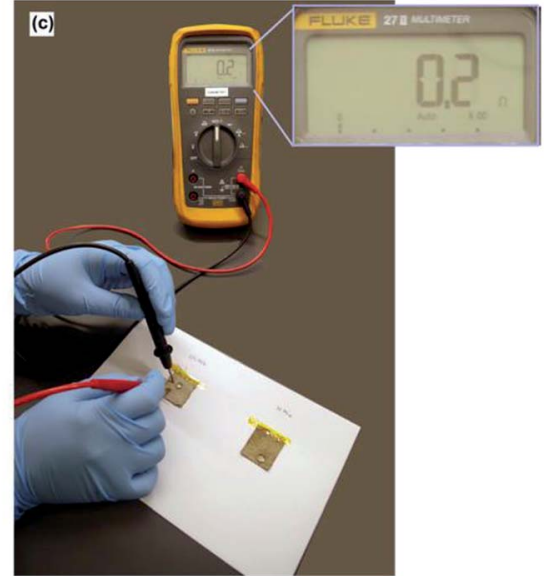

Fig. 4 (a) Water droplet on PFDA coated Ag-cotton (b) contact angle measurement and (c) surface resistance measurement.

conformal PFDA coating was applied, the surface sheet resistance of the water-repellent conductive cotton remained low $\left(0.2 \Omega \mathrm{cm}^{-1}\right.$, Fig. $\left.4 \mathrm{c}\right)$ even after encapsulation with an insulating polymer.

To test the chemical stability of the silver-coated cloths, Agcotton and PFDA-Ag-cotton were continuously exposed to air and moisture for one month. As seen in Fig. 5a and b, samples lacking the PFDA encapsulation turned black, suggesting that the silver coating was oxidized into silver oxide. In contrast, the PFDA encapsulated silver cloths remained silver-grey, similar to freshly-prepared samples. Next, the surface chemical composition and valence state of freshly-prepared Ag-cotton, Ag-cotton (exposed to air) and PFDA-Ag-cotton (exposed to air) were investigated using X-ray photoelectron spectroscopy (XPS). As seen in Fig. $5 \mathrm{c}-\mathrm{e}$, the $\mathrm{Ag} 3 \mathrm{~d}_{3 / 2}$ and $\mathrm{Ag} 3 \mathrm{~d}_{5 / 2}$ peaks for all samples appeared at 374.3 and $368.3 \mathrm{eV}$, respectively. The characteristic spin-orbit separation of $6.0 \mathrm{eV}$ confirmed that the peaks arose from bulk metallic silver. ${ }^{25,26}$ Deconvolution of these $\mathrm{Ag} 3 \mathrm{~d}$ peaks provided insight into the presence of oxidized silver species. After exposure to air, silver-coated cotton exhibited new $\mathrm{Ag} 3 \mathrm{~d}_{5 / 2}$ peaks at $367.8 \mathrm{eV}$ (Fig. $5 \mathrm{~d}$ ). The lower binding energy, as compared to $\mathrm{Ag}(0)$, indicated the presence of $\mathrm{Ag}_{2} \mathrm{O}^{27-29}$ However, the same $\mathrm{Ag} 3 \mathrm{~d}_{5 / 2}$ peak of air-exposed PFDA-Ag-cotton did not display any silver oxide peaks, suggesting that the PFDA layer prevented oxidation of the silver coating. In addition, the $\mathrm{O}$ 1s spectrum of Ag-cotton (exposed to air) (Fig. 5g) exhibited a small peak at $529.2 \mathrm{eV}$, consistent with $\mathrm{Ag}_{2} \mathrm{O}$ formation. ${ }^{29,30}$

The wash stability of PFDA-Ag-cotton was evaluated using AATCC test method 61, condition 2A, in which one accelerated laundering cycle is equivalent to five home or commercial laundering operations. ${ }^{31,32}$ Fig. 6 reveals that the apparent water contact angle of the rough samples minimally reduced with increasing laundering cycles. After four accelerated laundering cycles, the apparent water contact angle was $101^{\circ}$, which

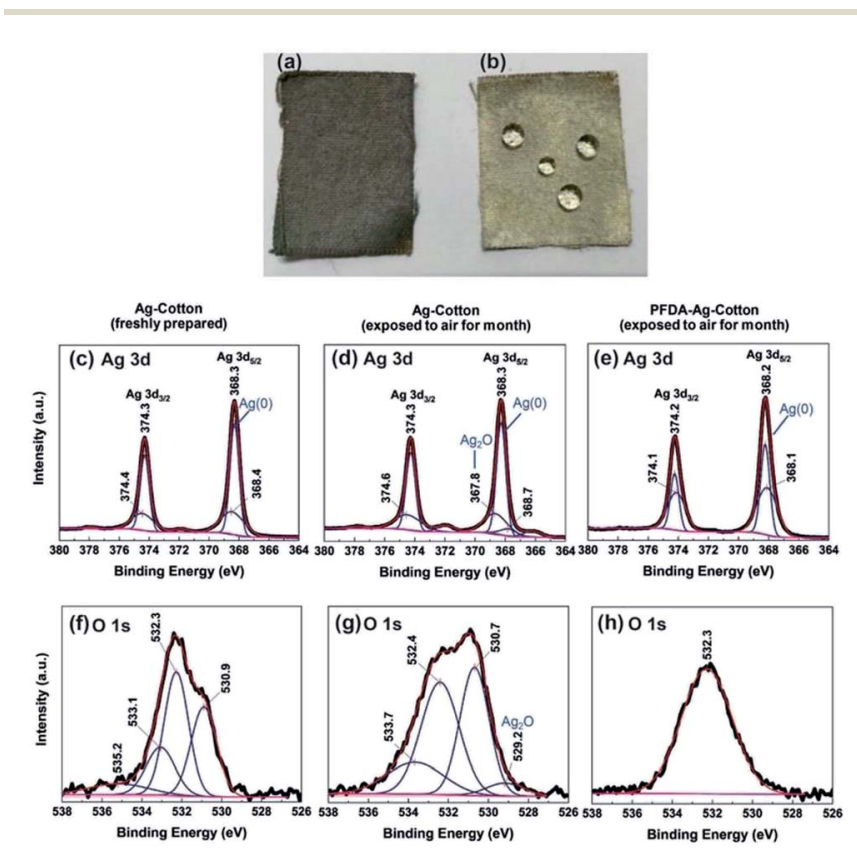

Fig. 5 (a) Optical image of silver-coated cotton after exposure to air. (b) Optical image of PFDA-encapsulated Ag-cotton after exposure to air. (c) Ag 3d peaks of freshly-prepared silver-coated cotton and (d) those of silver-coated cotton samples exposed to air. (e) $\mathrm{Ag} 3 \mathrm{~d}$ of PFDA-encapsulated Ag-cotton after exposed to air. (f) $O$ 1s peak of freshly-prepared silver-coated cotton and (g) that of silver-coated cotton samples exposed to air. (h) $\bigcirc$ 1s peak of PFDA-encapsulated Ag-cotton after exposure to air. 


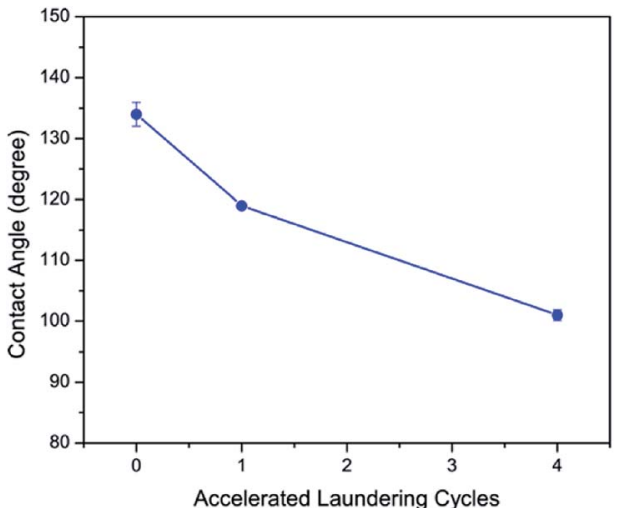

Fig. 6 Evolution of apparent water contact angle of PFDA-Ag-cotton with accelerated laundering cycles.

suggested that PFDA-Ag-cotton is washable and that the PFDA encapsulation layer could mechanically withstand up to twenty home laundering cycles.

\subsection{PFDA-Ag-cotton electrodes for triboelectric generator}

The PFDA-encapsulated, silver-coated cotton (PFDA-Ag-cotton) was used as top and bottom electrodes for a planar, face-on triboelectric device. Cotton functionalized with a perfluoroalkylated siloxane (F-cotton) was used a negative surface charge layer and was sewn onto PFDA-Ag-cotton. To create the complementary positive surface charge electrode, poly(dimethylsiloxane) (PDMS) was directly spray coated onto PFDAAg-cotton. According to the triboelectric series, ${ }^{33}$ PDMS is a positive triboelectric material in comparison to fluorinated materials. The positive and negative surface charge electrodes were then sewn together face-to-face to create the TEG (Fig. 7).

When the TEG was cyclically tapped with two fingers, the Fcotton and PDMS layers were brought into contact and released. Upon contact, negative surface charges from the F-cotton layer could be transferred to the PDMS surface, thus creating a compensating inductive current through the conductive PFDA-Ag-cotton electrodes. Upon release, an inductive current in the opposite direction should return the surface charge of each layer to their equilibrium condition. If the TEG is held at open circuit by connecting it to a $500 \mathrm{GOhm}$ load resistor, the open circuit voltage generated with tapping motions could be recorded. As seen in Fig. 8, a cycle of tapping produced an AC voltage (open circuit) and current (short circuit) output. A maximum open circuit voltage of $25 \mathrm{~V}$ and a maximum short circuit current of $2.25 \mu \mathrm{A}$ was observed. These figures of merit

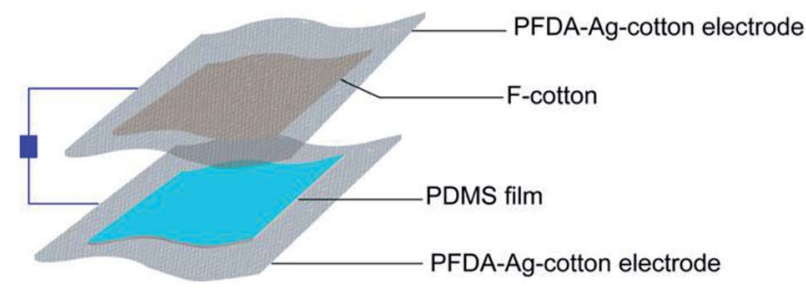

Fig. 7 Illustration of TEG device. (a)

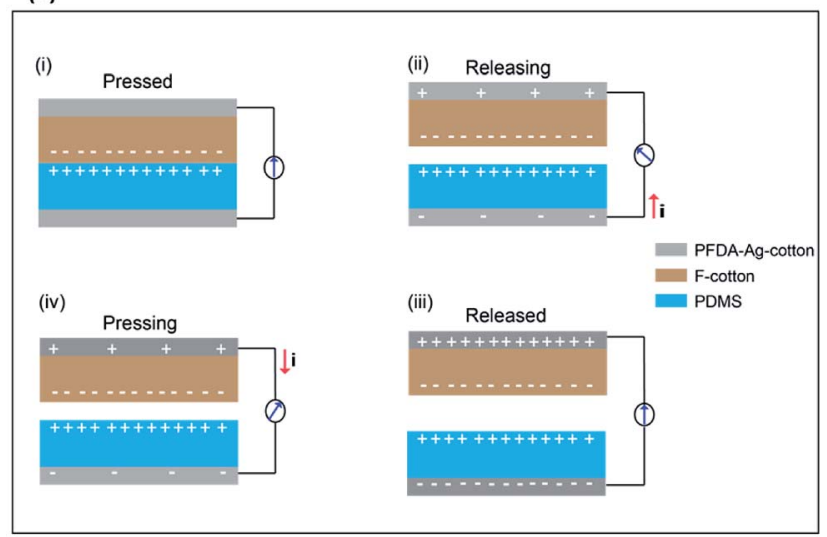

(b)
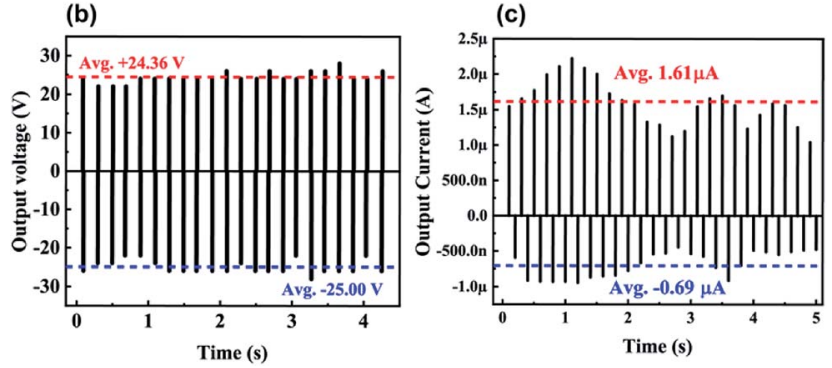

Fig. 8 (a) Schematic presentation of the working mechanism of the TEG and its (b) output voltage (c) and output current.

were comparable to previously-reported fabric patch devices, ${ }^{18,34,35}$ confirming that the PFDA-Ag-cotton electrodes reported here were comparable in electronic performance to known conductive fabrics.

\section{Conclusions}

We described an approach to create water-repellant conductive cotton using a direct, two-step coating and encapsulation procedure. First, we performed a facile electroless deposition using Tollens' reagent. We showed that the silver coatings thus created on cotton exhibited notable electrical conductivity. Next, we encapsulated the silver coating with a conformal layer of poly(perfluorododecylacrylate) (PFDA) using solvent-free initiated chemical vapor deposition (iCVD). Encapsulated, silver coated cotton (PFDA-Ag cotton) retained a very low surface resistance of $0.2 \Omega \mathrm{cm}^{-1}$ while also being water repellent, which means that these conductive fabrics will survive hot, humid conditions. PFDAAg-cotton served as competent charge collecting electrodes in an all-fabric triboelectric generator, supporting the use of this conductive cotton fabrics as electrodes for wearable electronics.

\section{Conflicts of interest}

The authors declare no conflicts of interest.

\section{Acknowledgements}

S. W. acknowledges financial support provided by King Mongkut's University of Technology Thonburi through the "Skill 
Development Grant for Young Faculty 2017 and 2018”. The "Young Scientist Researchers Fund" provided by the National Science and Technology Development Agency, Thailand. W. S. was supported by the "KMUTT 55th Anniversary Commemorative Fund”. S. Z. H., K.-W. P. and T. L. A. gratefully acknowledge support by the National Science Foundation under CHEM MSN 1807743.

\section{References}

1 W. Zeng, L. Shu, Q. Li, S. Chen, F. Wang and X. M. Tao, Adv. Mater., 2014, 26, 5310-5336.

2 J. S. Heo, J. Eom, Y. H. Kim and S. K. Park, Small, 2018, 14, 1703034.

3 S. de Mulatier, M. Nasreldin, R. Delattre, M. Ramuz and T. Djenizian, Adv. Mater. Technol., 2018, 3, 1700320.

4 Q. Y. Huang, D. R. Wang and Z. J. Zheng, Adv. Energy Mater., 2016, 6, 1600783.

5 S. Majumder, T. Mondal and M. J. Deen, Sensors, 2017, 17, 130.

6 V. Kaushik, J. Lee, J. Hong, S. Lee, S. Lee, J. Seo, C. Mahata and T. Lee, Nanomaterials, 2015, 5, 1493-1531.

7 T. L. Andrew, L. S. Zhang, N. Y. Cheng, M. Baima, J. J. Kim, L. Allison and S. Hoxie, Acc. Chem. Res., 2018, 51, 850-859.

8 A. K. Yetisen, H. Qu, A. Manbachi, H. Butt, M. R. Dokmeci, J. P. Hinestroza, M. Skorobogatiy, A. Khademhosseini and S. H. Yun, ACS Nano, 2016, 10, 3042-3068.

9 S. C. Liu, M. J. Hu and J. Yang, J. Mater. Chem. C, 2016, 4, 1320-1325.

10 X. Cauchy, J. E. Klemberg-Sapieha and D. Therriault, ACS Appl. Mater. Interfaces, 2017, 9, 29010-29020.

11 H. Y. Chen, F. Liao, Z. Y. Yuan, X. R. Han and C. J. Xu, Mater. Lett., 2017, 196, 205-208.

12 C. Wang, C. Xiang, L. Tan, J. W. Lan, L. H. Peng, S. X. Jiang and R. H. Guo, RSC Adv., 2017, 7, 40452-40461.

13 M. Montazer and V. Allahyarzadeh, Ind. Eng. Chem. Res., 2013, 52, 8436-8444.

14 S. P. Mu, H. Y. Xie, W. Wang and D. Yu, Appl. Surf. Sci., 2015, 353, 608-614.

15 R. Ashayer-Soltani, C. Hunt and O. Thomas, Text. Res. J., 2016, 86, 1041-1049.

16 Y.-J. Tan, J. Li, Y. Gao, J. Li, S. Guo and M. Wang, Appl. Surf. Sci., 2018, 458, 236-244.
17 K. K. S. Lau and K. K. Gleason, Macromolecules, 2006, 39, 3688-3694.

18 L. S. Zhang, Y. H. Yu, G. P. Eyer, G. Q. Suo, L. A. Kozik, M. Fairbanks, X. D. Wang and T. L. Andrew, Adv. Mater. Technol., 2016, 1, 1600147.

19 B. Xue, P. Chen, Q. Hong, J. Y. Lin and K. L. Tan, J. Mater. Chem., 2001, 11, 2378-2381.

20 J. Li, J. W. Zhu and X. H. Liu, Dalton Trans., 2014, 43, 132137.

21 B. Ameduri, B. Boutevin and G. Kostov, Prog. Polym. Sci., 2001, 26, 105-187.

22 A. E. Maher, C. M. Ambler, E. S. Powell and H. R. Allcock, J. Appl. Polym. Sci., 2004, 92, 2569-2576.

23 A. B. Lopez, J. C. de la Cal and J. M. Asua, Langmuir, 2016, 32, 7459-7466.

24 Z. Cui, E. Drioli and Y. M. Lee, Prog. Polym. Sci., 2014, 39, 164-198.

25 Z. Li, S. Z. Jiang, Y. Y. Huo, T. Y. Ning, A. H. Liu, C. Zhang, Y. He, M. H. Wang, C. H. Li and B. Y. Man, Nanoscale, 2018, 10, 5897-5905.

26 A. A. Mosquera, J. M. Albella, V. Navarro, D. Bhattacharyya and J. L. Endrino, Sci. Rep., 2016, 6, 32171.

27 H. Zhang, G. Wang, D. Chen, X. J. Lv and U. H. Jinghong, Chem. Mater., 2008, 20, 6543-6549.

28 Y. Han, R. Lupitskyy, T. M. Chou, C. M. Stafford, H. Du and S. Sukhishvili, Anal. Chem., 2011, 83, 5873-5880.

29 T. C. Kaspar, T. Droubay, S. A. Chambers and P. S. Bagus, J. Phys. Chem. C, 2010, 114, 21562-21571.

30 T. E. Jones, T. C. R. Rocha, A. Knop-Gericke, C. Stampfl, R. Schlogl and S. Piccinin, Phys. Chem. Chem. Phys., 2015, 17, 9288-9312.

31 B. Deng, R. Cai, Y. Yu, H. Q. Jiang, C. L. Wang, J. A. Li, L. F. Li, M. Yu, J. Y. Li, L. D. Xie, Q. Huang and C. H. Fan, Adv. Mater., 2010, 22, 5473-5477.

32 H. Z. Liu, M. Lv, B. Deng, J. Y. Li, M. Yu, Q. Huang and C. H. Fan, Sci. Rep., 2014, 4, 5920.

33 Z. L. Wang, ACS Nano, 2013, 7, 9533-9557.

34 W. Paosangthong, R. Torah and S. Beeby, Nano Energy, 2019, 55, 401-423.

35 A. Chandrasekhar, N. R. Alluri, B. Saravanakumar, S. Selvarajan and S. J. Kim, J. Mater. Chem. C, 2017, 5, 1810-1815. 